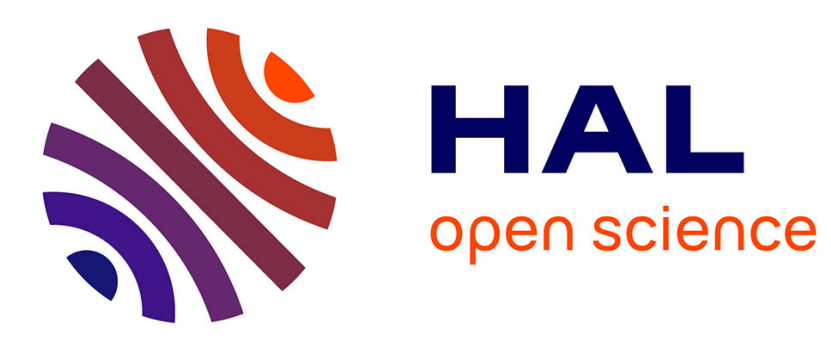

\title{
Transformer la participation de l'enfant aux activités de consommation alimentaire
}

\author{
Valérie-Inès de La Ville, Valérie Tartas
}

\section{To cite this version:}

Valérie-Inès de La Ville, Valérie Tartas. Transformer la participation de l'enfant aux activités de consommation alimentaire. Enfance, 2008, L'enfant face aux aliments, 60 (3), pp.299-307. 10.3917/enf.603.0299 . hal-01844096

\section{HAL Id: hal-01844096 https://hal.science/hal-01844096}

Submitted on 8 Mar 2019

HAL is a multi-disciplinary open access archive for the deposit and dissemination of scientific research documents, whether they are published or not. The documents may come from teaching and research institutions in France or abroad, or from public or private research centers.
L'archive ouverte pluridisciplinaire HAL, est destinée au dépôt et à la diffusion de documents scientifiques de niveau recherche, publiés ou non, émanant des établissements d'enseignement et de recherche français ou étrangers, des laboratoires publics ou privés. 


\title{
Transformer la participation de l'enfant aux activités de consommation alimentaire ${ }^{1}$
}

\author{
Valérie-Inés de La VILLE $^{2}$ et Valérie TARTAS ${ }^{3}$
}

\section{RESUME :}

Cet article discute les limites de deux modèles de socialisation économique largement utilisés dans la recherche en marketing - le modèle piagétien en stades de développement et celui du processus d'acquisition de comportements économiques. Pour étudier comment les enfants intègrent la culture de consommation de masse, il est nécessaire de considérer les apports de la psychologie culturelle qui révèle l'enjeu clé de la transformation de la participation de l'enfant à différentes activités conjointes de consommation. Comment l'enfant passe-t-il d'une participation périphérique (utilisateur d'un produit acheté par ses parents, observateur de situations d'achat) à une participation centrale (décideur sur certaines catégories d'aliments, expert reconnu de certains types de produits)? Cette approche éclaire les défis inhérents au marketing agro-alimentaire : d'un côté, il peut aider à surmonter les comportements néophobiques de l'enfant; de l'autre, en instituant l'enfant comme décideur de ses pratiques alimentaires, il est porteur de risques de transgression alimentaire ou de pratiques alimentaires détachées des normes diététiques.

Mots clés: Enfant consommateur, Psychologie culturelle, Socialisation économique, Marketing agroalimentaire.

\section{Transforming children's participation to consumption activities}

\section{SUMMARY :}

This paper discusses some weaknesses of two frameworks largely implemented in marketing research to describe children's economic socialization: a stage-based Piagetian perspective, and a process-centered model aiming to explain how children acquire economic behaviours. Studying the

\footnotetext{
${ }^{1}$ Cette recherche bénéficie du soutien de l'Agence Nationale de la Recherche dans le cadre du Programme National de Recherche sur l'Alimentation

2 Professeur de sciences de gestion à l'Université de Clermont 1. Coordinatrice du contrat ANR 'Ludo-Aliment' pour le CEREGE de l'Université de Poitiers. Centre Européen des Produits de l'Enfant, 186, rue de Bordeaux, 16000 Angoulême. delaville@iae.univ-poitiers.fr

${ }^{3}$ Maître de Conférences en psychologie du développement à l'Université Toulouse 2, Unité de Recherche Interdisciplinaire Octogone, E.A .4156, Laboratoire Cognition Communication et Développement. Pavillon de la recherche, 5 allée Antonio Machado 31058 Toulouse cedex 09. tartas@univ-tlse2.fr
} 
way children develop within contemporary mass consumption culture entails a shift towards cultural psychology theory which helps to focus on a key stake: the transformation of children's participation in different joint consumption activities. How do children move from a peripheral participation (as users of a product bought by parents, as observers of buying situations) to a central participation (as decision-makers or recognized experts on some categories of foods)? This approach highlights critical challenges that food marketing faces: on the one hand, it can help to overcome children's neophobic behaviour; on the other hand, it may encourage children's own food choices, hence introducing risks in terms of food transgression or new eating habits dissociated from healthy eating norms.

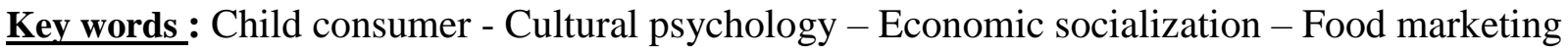

\section{INTRODUCTION}

La socialisation alimentaire du jeune consommateur est une question d'une complexité considérable. Nous proposons une analyse critique des perspectives utilisées dans la recherche en marketing pour modéliser le processus de socialisation des enfants en tant que consommateurs. Pour pallier certaines limites inhérentes à ces approches, nous mobilisons quelques acquis de la psychologie culturelle afin d'étudier la façon dont les jeunes mangeurs se réfèrent à la culture de consommation de masse. Nous esquissons les perspectives de recherche en marketing que ce cadre théorique ouvre.

\section{Affiner la modélisation de la socialisation économique grâce aux apports de la psychologie} culturelle

Pour analyser la socialisation économique de l'enfant, une première série de recherches en marketing utilise la théorie piagétienne appliquée au développement de la pensée économique. Une synthèse de ces modèles classiques a été présentée par Roedder-John (1999). Un second courant tente de pallier certaines limites de cette première approche en intégrant les aspects sociaux du développement économique. Toutefois, cette seconde approche dichotomise les dimensions sociales et individuelles dans l'explication de la socialisation économique. C'est pourquoi, une approche culturelle de la socialisation économique de l'enfant nous paraît fondée. 


\subsection{Limites de deux modèles classiques de la socialisation économique de l'enfant}

Nous développons brièvement l'approche proposée par Roedder-John dont nous avons déjà approfondi l'analyse (De La Ville \& Tartas, 2005). S'appuyant sur Piaget, elle détermine 3 stades définis en termes d'âge : perceptuel (3-7 ans), analytique (7-11 ans) et réfléchi (11-16 ans). Cette réduction des compétences cognitives et sociocognitives à des âges procède toutefois d'un détournement des thèses piagétiennes, Piaget ayant conceptualisé les stades de façon complexe en faisant référence aux mécanismes d'assimilation, d'accommodation et d'équilibration. Cette modélisation en 3 stades constitue un outil de synthèse destiné aux responsables marketing plutôt qu'une description fine des connaissances mobilisées par les enfants contemporains en situation de consommation. Les critiques habituellement adressées au modèle piagétien s'appliquent à ce modèle de socialisation : l'enfant considéré est davantage un sujet épistémique - et non un sujet social - ce qui conduit à souligner l'immaturité des compétences enfantines, en les décrivant d'abord comme uniquement perceptives, puis égocentriques, et n'étant susceptibles de déboucher sur des modalités plus critiques qu'à l'adolescence. Or les enfants contemporains, qui baignent dans la culture consumériste, acquièrent des connaissances et des modes de pensée extrêmement différents de ceux présumés par ces recherches qui appliquent le modèle de l'adulte raisonneur et logique comme aboutissement du développement. Cram \& Ng (1999) regrettent que l'influence des processus d'interactions sociales, des contextes historiques, des attitudes politiques et sociales, du rôle des habitudes et des valeurs sur le développement de la socialisation économique de l'enfant aient été négligés. Ces critiques s'appliquent au modèle de Roedder-John, dans lequel le rôle d'autrui est réduit à un facteur d'influence sur le développement des compétences de consommation, et qui écarte la possibilité que le rôle d'autrui, et plus largement de la culture, puisse être constitutif du développement. Ce modèle recèle 3 limites majeures : 1) l'enfant y est essentiellement décrit du point de vue de ses «incompétences », ses compétences en cognition sociale sont ainsi absentes de la littérature marketing sur la compréhension du mensonge ou du raisonnement d'autrui. (Deleau, 1999); 2) seuls les facteurs internes rendent compte d'un développement linéaire en stades, ce qui 
conduit à retenir l'âge comme facteur explicatif aux dépens des dimensions sociales, et à négliger les différences qualitatives dans la nature du raisonnement à chaque stade; 3) seul le mode logique ou paradigmatique est observé pour expliquer l'apprentissage des conduites de consommation, or les travaux de Jérôme Bruner (1986) ont démontré que le mode narratif constitue une voie d'entrée dans la culture tout à fait essentielle.

Une autre série de travaux en marketing met au contraire l'accent sur les facteurs sociaux (Moschis \& Churchill, 1978) et sur les processus permettant à l'enfant d'acquérir des compétences économiques. Lassare et Roland-Lévy (1989) prennent ainsi en compte 4 variables: les agents de socialisation (pairs, famille, école, mass media) ; les processus d'apprentissage (essentiellement l'imitation), les variables sociales structurales (âge, sexe, classes sociales) et le contenu d'apprentissage. Ce modèle réalise une synthèse entre 3 approches distinctes : 1) la théorie piagétienne, reprise par Berti et Bombi (1989) pour le développement de la pensée économique pointant le rôle actif de l'enfant ; 2) le modèle des rôles sociaux considèrant que l'enfant apprend différents rôles sociaux, dont celui de consommateur; 3) la théorie de l'apprentissage social de Bandura (1977) retenant 2 processus centraux (l'observation et l'imitation des parents, pairs, fratrie) pour expliquer les comportements de consommation concrets (comparer des prix et produits) et des notions plus complexes (pouvoir d'achat, taxes). L'environnement social est alors représenté par 2 variables: l'environnement social de l'enfant et la nature des feed-backs issus de ce même environnement. Bien que le rôle d'autrui y soit vu comme central, ces modèles n'étudient pas les interactions sociales car l'unité d'analyse reste l'individu. Deux limites majeures apparaissent : 1) ces études, focalisées sur les pré-adolescents ou adolescents, négligent d'étudier les enfants plus jeunes ; 2) l'observation et l'imitation, processus explicatifs de la dynamique de socialisation économique, peinent à décrypter l'émergence de comportements créatifs différents de ceux élaborés par l'entourage et n'expliquent pas ce qui se passe si les enfants sont confrontés à des comportements moins compétents. 


\subsection{Une intégration de la psychologie culturelle pour une reformulation de la problématique}

La prise en compte des actions des enfants en situation de consommation, c'est-à-dire des conduites «situées » et «distribuées » au sein de contextes communicatifs, apparaît comme une condition clé pour mieux comprendre comment l'enfant intègre la culture consumériste. En reprenant des éléments des théories attribuant au social - les autres ainsi que les instruments de la culture (Vygotski, 1934) un rôle constitutif dans le développement de l'enfant, nous esquissons les bases d'une approche culturelle de la socialisation économique de l'enfant. L'enjeu ne consiste plus alors à cerner une relation directe entre l'enfant et le produit en termes de préférences du jeune consommateur, ni à décrire comment certains facteurs (la famille, les médias, les pairs, l'école) influencent son comportement de consommateur. Le développement de la consommation alimentaire chez l'enfant doit prendre en compte 3 dimensions: 1) l'enfant n'est jamais seul face à un aliment : il est toujours guidé dans ses découvertes alimentaires par autrui (ses parents, sa fratrie, ses copains) ; 2) l'intérêt que porte un enfant à un produit alimentaire n'est jamais inné (Chiva, 1985): son lien à l'aliment n'est pas dû aux seules propriétés gustatives ou organoleptiques, mais aussi à 3) l'histoire sociale et institutionnelle de cet aliment, aux occasions de sa consommation et à l'imaginaire culturel qui lui sont associés dans une société donnée. L'accès ou l'interdit lié à certains aliments constitue un marqueur de rites sociaux ou religieux (anniversaire, puberté, communion, majorité, grossesse, pratique d'un art tel le Sumo) : la signification et la valeur de l'aliment se révèlent à travers les règles culturelles, sociales et institutionnelles de son usage. Même si la consommation alimentaire de l'enfant paraît solitaire par un accès autonome au réfrigérateur familial ou des dépenses d'argent de poche, il s'agit bien d'une activité sociale.

Dans ce cadre, la problématique de la socialisation alimentaire du jeune consommateur se pose en des termes plus larges : pour donner du sens à ses pratiques alimentaires et les faire évoluer, quel usage l'enfant fait-il des outils culturels qu'on lui a transmis ? Pour Rogoff et ses collaborateurs (2007), étudier la participation enfantine aux activités culturelles suppose de rendre compte à la fois du rôle de guide des traditions culturelles et du rôle actif des enfants eux-mêmes. Ce qui centre 
l'analyse sur l'effet combiné des médiateurs culturels qui structurent l'expérience alimentaire de l'enfant: le langage, le cercle familial, le groupe de pairs, les systèmes techniques et médiatiques auxquels l'enfant a accès, les techniques marketing mobilisées par les entreprises. Cette perspective permet de poser d'autres questions sur la socialisation économique de l'enfant. Quels sont les médiateurs culturels à l'œuvre lorsqu'un enfant reconnaît un aliment comme lui étant destiné ? De quelle façon un enfant apprend-il à faire un usage socialement acceptable d'un aliment en distinguant les différents contextes de son usage? Quels instruments culturels les enfants mobilisent-ils pour effectuer des arbitrages concernant des décisions d'achat ou de consommation d'aliments? Comment l'enfant va-t-il prendre en compte les contextes pour négocier, c'est-à-dire adapter la formulation et la justification de sa demande (friandise comme récompense de résultats scolaires, aliment comme expression de lien affectif, aliment présenté comme sain). La perspective culturelle vise à comprendre comment l'enfant devient capable de discriminer les contextes de signification dans lesquels se déroule l'activité conjointe de consommation, de distinguer les pratiques auxquelles il doit se référer pour ajuster son niveau d'engagement et valorise la capacité créative de l'enfant qui joue avec les règles imposées, parfois en les détournant. La notion de participation de l'enfant à des activités conjointes de consommation souligne le rôle clé d'autrui et de la culture de consommation de masse contemporaine pour le développement des comportements économiques de l'enfant tout en intégrant l'évolution progressive des éléments institutionnels qui légitiment la place de l'enfant au cœur des relations marchandes.

\section{Ambivalence de la visée transformatrice du marketing agro-alimentaire ciblant les enfants}

Dans ce cadre théorique, les techniques marketing (design, packaging, communication publicitaire, merchandising, animation du point de vente) sont des médiateurs culturels qui structurent la participation de l'enfant à des activités conjointes de consommation alimentaire. La participation du nourrisson ou du jeune enfant est indirecte: ses parents font leurs courses en fonction de ses préférences et des découvertes gustatives qu'ils souhaitent lui procurer. Quand l'enfant accompagne ses parents dans les magasins, il découvre l'univers des mises en scènes commerciales, apprend à 
faire un usage approprié de notions (marque, prix, qualité) qu'il exploite pour influencer les décisions. Quand les médiateurs marketing lui permettent de saisir lui-même les produits, l'enfant est incité à agir tel un consommateur adulte. Le rôle du marketing consiste à accompagner et susciter la transformation de la participation de l'enfant aux activités conjointes de consommation. Comment l'enfant passe-t-il d'une participation périphérique - utilisateur d'un produit acheté par ses parents, observateur de situations d'achat - à une participation centrale - décideur et expert reconnu sur certaines catégories d'aliments? Ce processus de socialisation économique transforme les normes selon lesquelles la participation de l'enfant aux activités conjointes de consommation alimentaire est jugée acceptable dans les diverses communautés sociales auxquelles l'enfant se réfère et qui lui accordent un pouvoir de décision plus ou moins étendu sur ses propres pratiques (Lave \& Wenger, 1991).

\subsection{Le marketing agro-alimentaire comme facteur de risque alimentaire}

Dans un contexte d'industrialisation accrue des aliments et de pandémie d'obésité, les pratiques marketing de l'industrie agro-alimentaire font l'objet de vives critiques de la part de journalistes, d'associations de consommateurs et de chercheurs (Poulain, 2002). Les enfants apparaissent comme «des canaris dans la mine de charbon», la population la plus exposée à cette alimentation industrielle et aux techniques de persuasion qui l'accompagnent (Kline, 2005). Comment interpréter l'émergence d'un segment d'aliments ludiques, produits pour lesquels les médiateurs marketing renvoient à l'univers enfantin, ses plaisirs, ses références en termes de héros, de modes ? L'enfant reconnaît un aliment comme lui étant destiné grâce à sa forme qui reprend la silhouette d'un héros de bande dessinée, à la présence d'une mascotte ou du logotype de la marque apposés sur l'emballage, à une évocation gustative décalée ou plus ou moins transgressive, ou par le caractère ludique de la narration publicitaire associée au produit. La remise en cause des modes de catégorisation habituels des produits, l'insertion des produits alimentaires dans l'actualité des cultures enfantines, le « cobranding » entre marques alimentaires et marques de jeux et jouets, des formes de packaging permettant des usages régressifs et nomades, sont autant de techniques visant à créer un décalage 
faisant référence à une activité située au-delà de l'acte alimentaire. L'aliment est consommé pour ce qu'il représente, ce qu'il permet en matière d'interactions sociales avec les pairs et l'autonomie qu'il procure à l'enfant. Le rajout d'une dimension ludique à l'aliment, en renforçant les médiations marketing qui mettent en exergue les aspects hédoniques de la consommation alimentaire, serait l'expression la plus aboutie de l'intérêt bien compris des industriels pour attirer, séduire et fidéliser l'enfant. Car poussée à son paroxysme, cette modernité créée et exploitée par le marketing conduirait à des formes d'autonomisation des pratiques alimentaires des enfants en-dehors du contrôle parental ou adulte et contribuerait à renforcer la situation d'anomie dans laquelle se trouve l'enfant mangeur contemporain (Fischler, 1979). En effet, l'enfant est habituellement décideur de ses pratiques alimentaires dans le cadre d'activités considérées comme des espaces propres de l'enfance dans la société contemporaine : pour son anniversaire, il choisit les confiseries et les boissons qui vont être offerts ; pour ses goûters à l'école, il décide quels produits échanger avec ses amis ; pour d'autres occasions extraordinaires au sens étymologique - une pyjama partie ou une soirée télé entre amis -, l'enfant pourra inclure des aliments présentant des formes de transgression tolérées. Toutefois, la situation change lorsque les médiateurs marketing incitent l'enfant à définir ses propres pratiques alimentaires dans le quotidien, en fonction de critères ou de règles qui sont déconnectées des traditions familiales ou détachées des normes diététiques prônées par les éducateurs. C'est bien à travers ce type d'action que le marketing constitue un facteur de risque car il inculque à l'enfant d'autres raisons ou d'autres règles pour manger, sur lesquelles les éducateurs ont peu de prise. L'enfant se retrouve alors confronté à une série d'injonctions contradictoires entre le discours familial de transmission sociale et culturelle, le discours nutritionnel imposé dans le cadre de l'institution scolaire et le discours de nature hédoniste que lui adressent les médiateurs marketing (Buijzen et al., 2008). Perdu par des explications pseudo-scientifiques quant aux effets à court et long terme des nutriments sur sa santé, renvoyant dos à dos des argumentations contradictoires afin de se donner des marges de manœuvre, l'enfant développe un comportement alimentaire qui ne semble plus maîtrisable dans le cadre d'une régulation sociale et culturelle traditionnelle. Selon l'horizon 
temporel pris en compte, les médiateurs marketing peuvent ainsi contribuer à accroître l'anxiété au plan individuel mais aussi le risque sociétal au plan collectif.

\subsection{Le marketing agro-alimentaire comme médiation éducative}

La situation de l'enfant mangeur contemporain peut être aussi analysée d'un autre point de vue, conduisant à d'autres pratiques marketing. Comprenant que l'enfant est effectivement soumis à des discours contradictoires sur l'alimentation, certaines entreprises mettent en place des médiateurs marketing qui tiennent compte de la nécessité d'instaurer une régulation des prises alimentaires de l'enfant. Ces entreprises ne dirigent pas directement leur communication vers l'enfant, mais davantage vers l'adulte, l'éducateur, le parent ou l'enfant plus expert pour contribuer à inculquer des habitudes alimentaires en fonction du contexte social et institutionnel de son développement. Dans cette perspective, l'émergence d'aliments ludiques peut être vue comme une opportunité puisque de nombreux parents d'enfants de 5 à 7 ans - enfants majoritairement néophobiques (Pliner, 1994) utilisent le jeu pour leur faire accepter un aliment nouveau. L'introduction d'une dimension ludique vient alors en appui des efforts éducatifs des parents pour élargir le répertoire gustatif de leurs enfants et leur inculquer certaines valeurs liées aux occasions de consommation des différents aliments. Certaines entreprises créent de la valeur en apportant des solutions inédites pour pallier des vides institutionnels en matière alimentaire. L'absence de cantines dans la plupart des établissements scolaires aux Etats-Unis pousse les parents à composer un déjeuner avec des sodas et des produits faciles à grignoter mais présentant de trop fortes teneurs en sucre, sel et graisses. Constatant que la majorité des enfants américains mange très rarement des légumes et des produits frais, une jeune société ${ }^{4}$ fournit une lunch-box contenant des produits issus de l'agriculture biologique : un sandwich élaboré avec des légumes frais ayant une forme amusante de main ou de visage souriant, un dessert de fruits frais et une bouteille d'eau. En pratiquant un marketing sensoriel qui s'adresse directement à l'enfant et en apportant une solution institutionnelle qui réaffirme le rôle éducatif des parents en matière d'alimentation, cette entreprise se propose de transformer la participation de l'enfant à des

\footnotetext{
${ }^{4}$ Site: http://www.kidfresh.com
} 
activités conjointes de consommation avec une visée clairement éducative. Dans un contexte de crise alimentaire permanente, d'anxiété croissante concernant les produits de l'industrie agro-alimentaire, il devient pertinent et rentable d'élaborer un marketing éducatif qui contribue à réguler le comportement alimentaire des enfants en les aidant à maîtriser les tensions entre compulsion et satiété, gourmandise et nutrition, banalité des saveurs et extension de leur répertoire gustatif. Cette perspective permet de tenir compte de l'inventivité des pratiques enfantines et de formes de créativité collective qui émergent de l'évolution permanente des cultures enfantines (Sirota, 1998). En contribuant à transformer les comportements individuels de consommation, le marketing peut espérer revendiquer une part constructive dans l'éducation du jeune consommateur, comme le démontre l'initiative menée avec succès depuis plus de 30 ans en Italie de façon conjointe par le Ministère de l'Education Nationale et le réseau des coopératives de distribution (Drasigh, 2004). Loin d'un «laisser-faire » teinté d'un relent d'idéologie néo-libérale mal maitrisée, le marketing agroalimentaire ne peut plus faire l'économie d'un questionnement approfondi sur les responsabilités morales et éthiques qui sont les siennes dans les sociétés occidentales ou dans les sociétés en développement (Bergadaa, 2004). Or ce débat est occulté dans le cadre d'une idéologie positive de la science qui prétend dire le vrai, mais refuse de se penser comme responsable des transformations qu'elle rend possibles ou signifiantes.

\section{Conclusion}

Nous avons montré que des discours contradictoires sur les effets des techniques du marketing agroalimentaire s'arc-boutent soit sur la notion de préférences gustatives chez l'enfant, soit sur celle de programmation gustative créée par l'industrie. Mobilisant les acquis de la psychologie culturelle, nous avons remis en question ces deux notions afin de montrer que les techniques marketing n'ont pas de signification intrinsèque. L'effet délétère qu'elles produisent sur les règles du manger ou au contraire l'effort en matière d'éducation alimentaire qu'elles appuient dépendent de la visée des médiations marketing. Cette problématique met en lumière quelques défis auxquels est confronté le marketing agro-alimentaire qui s'inscrit dans un système culturel, institutionnel, social et politique 
complexe, entremêlant un ensemble ambivalent de logiques, d'intérêts et de systèmes de valeurs (De La Ville, 2007).

\section{RÉFÉRENCES}

Bandura, A. (1977). Social learning theory. Englewood Cliffs, NJ, Prentice Hall.

Bergadaa, M. (2004). Evolution de l'épistémè économique et sociale : proposition d'un cadre de morale, de déontologie, d'éthique et de responsabilité pour le marketer. Recherche et Applications en Marketing, 19, 55-72.

Berti A., \& Bombi, A.S (1988). The child's construction of economics. Cambridge, UK: Cambridge University Press.

Bruner, J. (1986/2000). Actual Minds, possible worlds. Cambridge, MA: Harvard University Press.

Buijzen, M., Schuurman, J., \& Bomhof, E. (2008). Associations between children's television advertising and their food consumption patterns: a household diary-survey study. Appetite, 50, 231-239.

Chiva, M. (1985). Le doux et l'amer. Sensation gustative, émotion et communication chez le jeune enfant. Paris: PUF.

Cram, F., \& Ng, S.H. (1999). Consumer Socialization. Applied Psychology: An international review, 48, 297-312.

De La Ville, V.I. (2007). The consequences and contradictions of child and teen consumption in contemporary practice. Society and Business Review, 2, 7-14.

De La Ville, V.I., \& Tartas V. (2005). L'activité de consommation enfantine et ses médiateurs. In V.I. De La Ville (Ed.), L'enfant consommateur (pp. 73-88). Paris: Vuibert.

Deleau M. (1999). Psychologie du développement. Paris : Bréal.

Drasigh, S. (2004). Les centres d'éducation à la consommation responsable : l'expérience des COOP en Italie. In N. Diasio (Ed.), Au Palais de Dame Tartine (pp. 197-204). Paris: L'Harmattan.

Fischler, C. (1979). Gastronomie et gastro-anomie: sagesse du corps et crise bioculturelle de l'alimentation moderne. Communications, 31, 189-210.

Kline, S. (2005). Comment transformer la consommation enfantine dans la société du risque ? In V.I De La Ville (Ed.), L'enfant consommateur (pp. 281-300). Paris: Vuibert. 
Lassare, D., \& Roland-Lévy, C. (1989). Understanding children's economic socialization. In K.G. Grünert \& F. Olander (Eds.), Understanding economic behaviour (pp.347-368). Dordrecht: Kluwer Academic Publishers.

Lave, J., \& Wenger E. (1991). Situated learning: Legitimate peripheral participation. Cambridge: Cambridge University Press.

Moschis, G., \& Churchill, G. (1978). Consumer socialization: a theoretical and empirical analysis. Journal of Marketing Research, 15, 599-609.

Pliner, P. (1994). Development of measures of food neophobia in children. Appetite, 23 (2), 147-163.

Poulain, J.P. (2002). Sociologies de l'alimentation. Paris: PUF..

Roedder-John, D. (1999). Consumer Socialization of Children - A Retrospective look at twenty-five years of research. Journal of Consumer Research, 26, 183-213.

Rogoff, B., Moore, L., Najafi B., Dexter, A., Correa-Chavez M., \& Solis, J. (2007). Développement des répertoires culturels et participation des enfants aux pratiques quotidiennes. In G. Brougère \& M. Vandenbroeck (Eds). Repenser l'éducation des jeunes enfants (pp.103-138), Bruxelles : P.I.E. Peter Lang

Sirota, R. (1998). Les copains d'abord. Les anniversaires de l'enfance, donner et recevoir, Ethnologie française, XXVIII, 457-471.

Vygotski, L.(1934/1985). Pensée et langage. Paris: Editions sociales. 